\title{
Erratum to: Web-Age Information Management (Part I and II)
}

\author{
Bin Cui ${ }^{1(\bowtie)}$, Nan Zhang ${ }^{2}$, Jianliang $\mathrm{Xu}^{3}$, Xiang Lian ${ }^{4}$, and Dexi Liu ${ }^{5}$ \\ ${ }^{1}$ Peking University, Beijing, China \\ 2 The George Washington University, Washington, D.C., USA \\ ${ }^{3}$ Hong Kong Baptist University, Kowloon Tong, Hong Kong, SAR China \\ ${ }^{4}$ University of Texas Rio Grande Valley, Edinburg, TX, USA \\ 5 Jiangxi University of Finance and Economics, Nanchang, China
}

\section{Erratum to: \\ B. Cui et al. (Eds.) \\ Web-Age Information Management (Part I and II) \\ DOI: $10.1007 / 978-3-319-39958-4$}

In an older version of the paper starting on page 441 of the first volume of the WAIM proceedings (LNCS 9658), the name and email address of the second author (Qin Liu) were missing. This has been corrected. Consequently, the Contents and the Author Index has also been updated in this volume (LNCS 9659).

In an older version of the paper starting on p. 521 of the WAIM proceedings (LNCS 9659), the corresponding author was given incorrectly. Jia Zhu is the correct corresponding author.

The updated original online version for this Book can be found at 10.1007/978-3-319-39958-4 